\section{P128 CONTEXTUAL APPROACHES TO UNDERSTANDING HIV TESTING AND PREVENTION ENGAGEMENT AMONG URBAN REFUGEE ADOLESCENTS AND YOUTH IN KAMPALA, UGANDA}

${ }^{1} \mathrm{C}$ Logie*, ${ }^{2} \mathrm{M}$ Okumu, ${ }^{3} \mathrm{R}$ Hakiza, ${ }^{4} \mathrm{~S}$ Mwima, ${ }^{5} \mathrm{P}$ Kyambadde, ${ }^{6} \mathrm{~L}$ Mbuagbaw. ${ }^{1}$ University of Toronto, Toronto, Canada; ${ }^{2}$ University of North Carolina Chapel Hill, Chapel Hill, USA; ${ }^{3}$ Young African Refugees for Integral Development, Kampala, Uganda; ${ }^{4}$ Uganda Ministry of Health, Kampala, Uganda; ${ }^{5}$ MARPI Clinic, Mulago Hospital, Kampala, Uganda; ${ }^{6}$ McMaster University, Hamilton, Canada

\subsection{6/sextrans-2021-sti.243}

Background HIV prevention needs are understudied with urban refugee youth. We explored experiences, preferences and engagement with HIV testing and prevention among urban refugee adolescents and youth in Kampala, Uganda, with a focus on the role of contextual factors in shaping access and uptake.

Methods This qualitative community-based study with urban refugee youth aged 16-24 living in Kampala's informal settlements involved five focus groups (FG), including two with women, two with men, and one with sex workers. We also conducted five in-depth key informant interviews. We conducted thematic analysis informed by Campbell and Cornish's conceptualization of material and symbolic contexts.

Results Refugee youth participants $(n=44$; mean age: 20.25, SD: 2.19 ; men: $n=17$; women: $n=27)$ were from the Democratic Republic of Congo $(n=29)$, Rwanda $(n=11)$, Burundi $(n=3)$, and Sudan $(n=1)$. Participant narratives reflected material, symbolic and relational contexts that shaped HIV testing awareness, preferences and uptake. Material contextual factors that presented barriers to HIV testing and prevention engagement included: transportation costs to clinics, overcrowded living conditions that limited access to private spaces, low literacy, and language barriers. Symbolic contexts that constrained HIV testing engagement included medical mistrust of HIV testing, and inequitable gender norms. Religion emerged as an opportunity to connect with refugee communities and to address conservative religious positions on HIV and sexual health. Relational contexts connected with HIV prevention and testing engagement included linkages with professional support, family, friends, and intimate partners. Many participants suggested the need for mental health support alongside HIV care, particularly for HIV testing.

Conclusion Efforts to increase access and uptake along the HIV testing and prevention cascade can meaningfully engage urban refugee adolescents and youth to develop culturally and contextually relevant services to optimize HIV and sexual health outcomes. Integrated mental health and HIV services may be warranted for urban refugee adolescents and youth.

\section{P130 TACKLING ANTIMICROBIAL RESISTANCE USING BIOINFORMATICS APPROACH IN SUPERBUG NEISSERIA GONORRHOEAE BY TARGETING GLUTAMATE RACEMASE (MURI)}

R Kant*, P Jha, M Chopra, D Saluja. University of Delhi, New Delhi, India

\subsection{6/sextrans-2021-sti.244}

Background Neisseria gonorrhoeae, a causative agent of gonorrhea, has developed resistance for most of the drugs and hence recently declared as 'Superbug'. Glutamate racemase (MurI)which is considered as an important drug target because it has shown an integral role in bacterial cell wall synthesis. Therefore, there is an intense need for identification of novel drugs for the treatment of gonorrhea.

Methods Based on the amino acid sequence of Neisseria gonorrhoeae MurI(YP_208550; Strain FA1090), a homology model was generated to perform homology modeling based on the PDB BLAST results and their validation based on DOPE score and PDF energy score which was further verified by Verify-3D protocol and Ramachandran Plot.co-crystalized ligand of the template was docked into the modeled MurI structure, after superimposition of template structure and modelled structure. Based on docking score, best pose was selected and receptor-ligand pharmacophore model was generated. Virtual screening of potent inhibitors against the pharmacophore model was performed, best hits were selected based on ADMET profile and further refined.

Results The best homology model generated was selected based on the verify score of 107.93 using Discovery Studio 4.0. Ramachandran plot showed 214 residues (91.8\%) fall in most favored region. Quality factor of $84 \%$ for the protein models was obtained using ERRAT. Six pharmacophores were generated using best docking pose between D-glutamate and MurI. These were subjected to virtual screening with ZINC database. 2214 hits were filtered by fit value of 1.5 which has resulted in 594 filtered hits. Further refinement done by subjecting these 594 hits to Lipinski and veber filter followed by ADMET, which finally gave 378 hits.

Conclusions The study identifies potential compounds that interact with active site of MurI protein, opening new avenues for the treatment option against multi-drug resistant strains of this pathogen.

\section{P131 CHLAMYDIA TRACHOMATIS INDUCES FERROPTOSIS TO PROMOTE ITS OWN DISSEMINATION BY INHIBITING SLC7A11/GPX4 SIGNALING}

${ }^{1} \mathrm{~W}$ Chen*, ${ }^{1} X$ Su, ${ }^{1} \mathrm{H}$ Luo, ${ }^{1} Z \mathrm{Z}$ Mai, ${ }^{2} X \mathrm{Xu},{ }^{1} \mathrm{~L}$ Zeng, ${ }^{1} Y$ Pan, ${ }^{1} Y$ Liao, ${ }^{2} \mathrm{Q} X \mathrm{Xu}^{3} \mathrm{~W}$ Zhao, ${ }^{3} \mathrm{~B}$ Zhang, ${ }^{1} \mathrm{Q}$ Xie, ${ }^{1} \mathrm{Y} X u{ }^{1}{ }^{1} \mathrm{H}$ Zheng. ${ }^{1}$ Dermatology Hospital, Southern Medical University, Guangzhou, China; ${ }^{2}$ Guangdong Dermatology Clinical College, Anhui Medical University, Hefei, China; ${ }^{3}$ Guangdong Provincial Key Laboratory of Tropical Disease Research, School of Public Health, Southern Medical University, Guangzhou, China

\subsection{6/sextrans-2021-sti.245}

Background Chlamydia trachomatis (C. trachomatis), an obligate intracellular bacterium, exits the host through lysis to reinitiate its life cycle in new hosts. However, the mechanism underlying lytic egress is not well understood.

Methods Propidium iodide staining and lactate dehydrogenase release assays were utilized to evaluate cell death during C. trachomatis infection. Lipid peroxide production, a hallmark of ferroptosis, was evaluated using the lipophilic fluorescent dye C11-BODIPY 581/591. Western blot and was employed for quantifying the ferroptosis-associated factors in the late stage of C. trachomatis infection. Ferroptosis inhibitors ferrostatin-1 and liproxstatin-1 were used to investigate the role of ferroptosis in C. trachomatis infection.

Results We found that lysis of C. trachomatis-infected cells in the late stage of infection did not involve apoptosis/necroptosis but occurred via ferroptosis, a recently described form of programmed cell death. Reduced levels of solute carrier family 7 member 11 (SLC7A11), glutathione, and glutathione peroxidase $4(\mathrm{GPx} 4)$, as well as accumulation of lipid peroxidation products, dysregulated cellular redox homeostasis in the late 
of C. trachomatis infectious cycle. Inhibiting lipid peroxidation using ferrostatin-1 and liproxstatin-1 not only abolished C. trachomatis-induced cell death, but also blocked C. trachomatis egress. Three C. trachomatis biovars were found to disseminate via ferroptosis, indicating that it is a conserved process. Conclusion C. trachomatis hijacks host cell machinery of ferroptosis in the late stage of infection for dissemination.

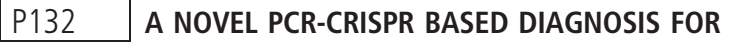 TREPONEMA PALLIDUM DETECTION, GENOTYPING, AND DRUG-RESISTANCE MUTATION IDENTIFICATION IN REAL-TIME}

W Chen*, H Luo, Z Mai, Y Jiang, Z Hu, L Zeng, H Zheng. Dermatology Hospital, Southern Medical University, Guangzhou, China

\subsection{6/sextrans-2021-sti.246}

Background The sexually transmitted disease syphilis caused by Treponema pallidum subsp. pallidum (TPA), is resurging worldwide, particularly in developing countries. Nucleic acid amplification tests (NAATs) possess highly sensitive and specifical capabilities, which have been largely utilized for pathogen detection, e. g. virus, bacteria, and fungus. However, the traditional NAATs applied to diagnose TPA are insufficiently sensitive.

Methods We established a novel PCR-CRISPR based syphilis molecular diagnosis with multiple excellent utilities.

Results The new approach retains the advantage of the high specificity of NAATs and allows detecting TPA with low concentrations at the single molecular level, which is more sensitive than the classical PCR assay. We also adapt this method to analyze the TPA by Nichols-/SS14-like cluster genotyping and macrolide resistance-associated mutations detection in realtime, providing a powerful tool for improving syphilis surveillance and clinical decisions/treatment selections. We further applied these assays to evaluate a panel of 42 TPA strains circulating in Southern China and find that the prevalent TPA strains consist of $83.33 \%$ SS14- and $16.67 \%$ Nichols-like TPA strains, with $97.67 \%$ of strains harboring $23 \mathrm{~s}$ rRNA mutations (A2058G or A2059G).

Conclusion We developed a novel PCR-CRISPR-based syphilis molecular assay, which exhibits robust capabilities and prospects in the diagnosis and molecular epidemiology of TPA.

\section{P135 HIGH PREVALENCE OF CURABLE SEXUALLY TRANSMITTED INFECTIONS AMONG 14-19 YEAR OLD ADOLESCENTS AT RISK OF HIV INFECTION IN KAMPALA, UGANDA}

${ }^{1} \mathrm{Y}$ Mayanja*, ${ }^{1} \mathrm{~J}$ Lunkuse, ${ }^{1} \mathrm{H}$ Kalungi, ${ }^{2} \mathrm{~W}$ Senyonga, ${ }^{1} \mathrm{O}$ Kamacooko, ${ }^{1} \mathrm{E}$ Ruzagira. ${ }^{1} \mathrm{MRC} /$ UVRI and LSHTM Uganda Research Unit, Entebbe, Uganda; ${ }^{2}$ Ministry of Health, Kampala, Uganda

\subsection{6/sextrans-2021-sti.247}

Background Sexually transmitted infections (STIs) are of public health significance; they have adverse reproductive health consequences and yet are preventable and curable. We studied STI prevalence and associated factors among adolescents in Kampala, Uganda.

Methods We conducted a cross-sectional study among 14-19year-old high-risk adolescents from March 2019 to March 2020. An interviewer-administered questionnaire was used to collect socio-demographic and behaviour data. STI tests were performed on urine using geneXpert for Chlamydia trachomatis (CT) and Neisseria gonorrhoeae (NG), and serum using the Rapid plasma reagin/Treponema Pallidum Particle Agglutination Assay for syphilis. Outcomes were positive tests for $\mathrm{CT}, \mathrm{NG}$, and/or active syphilis (titre $\geq 1: 8$ ). Statistical analysis was conducted using logistic regression.

Results We enrolled 490 volunteers with a median age of 18 years (Interquartile range 17-18 years). Most were female $(60.6 \%), \geq 18$ years $(50.8 \%)$, had only primary school education $(91.0 \%)$ and had their sexual debut at $<15$ years (48.4\%). Females reported a higher prevalence of paid sex than males both at the last sexual encounter $(96.4 \%$ vs $3.6 \%, \mathrm{p}<0.001)$ and in the past 3 months $(94.8 \%$ vs. $5.2 \%$, $\mathrm{p}<0.001) ; 128(26.1 \%)$ individuals reported change of residence in the past year with the frequency being higher in females than in males $(82 \%$ vs $18 \%, \mathrm{p}<0.001)$. STI prevalence was 39\% [CT (21.4\%), NG (5.9\%), active syphilis (1.4\%), multiple infections (10.3\%)] and higher among females than males $(75 \%$ vs $25 \% ; \mathrm{p}<0.001)$. Prevalent STIs were associated with paid sex at the last encounter (aOR 1.78 ; 95\% CI 1.02-3.16) and change of residence in the past year. The effect of changing residence was modified by sex; being significant for female (aOR 2.17; 95\% CI 1.293.67 ) but not male (aOR $0.26 ; 95 \%$ CI $0.33-2.00$ ) participants.

Conclusions Curable STIs are highly prevalent in this adolescent population. Interventions should increase access to STI prevention and treatment in sex work hotspots.

\section{P137 VIRAL HEPATITIS PREVALENCE AMONG MEN WHO HAVE SEX WITH MEN RECRUITED IN AN HIV PREP DEMONSTRATION STUDY IN COTONOU, BENIN}

\begin{abstract}
1,2,3 $\mathrm{L}$ Behanzin*, ${ }^{2,3} \mathrm{~F}$ Guédou, ${ }^{2} \mathrm{M}$ Goma, ${ }^{2} \mathrm{M}$ Olodo, ${ }^{2} \mathrm{M}$ Aza-Gnandji, ${ }^{2} \mathrm{~A}$ Dossouvo, ${ }^{2} \mathrm{~F}$ Kintin, ${ }^{4} \mathrm{~A}$ Akpaka, ${ }^{5} \mathrm{E}$ Chagas, ${ }^{6} \mathrm{~S}$ Ogoundélé, ${ }^{6} \mathrm{C}$ Ahouada, ${ }^{7,8,9} \mathrm{~F}$ Gangbo, ${ }^{8,9} \mathrm{M}$ Zannou, $3,10,11$ M Alary. ${ }^{1}$ Ecole Nationale de Formation des Techniciens Supérieurs en Santé Publique et en Surveillance Epidémiologique (ENATSE), Université de Parakou, Parakou, Bénin; ${ }^{2}$ Dispensaire IST, Centre de santé communal de Cotonou 1, Cotonou, Bénin; ${ }^{3}$ Axe Santé des populations et pratiques optimales en santé, Centre de recherche du CHU de Québec Université Laval, Québec, Canada; ${ }^{4}$ BENIN SYNERGIES PLUS (BeSyP), Cotonou, Bénin; ${ }^{5}$ RESEAU SIDA BENIN (RSB), Cotonou, Bénin; ${ }^{6}$ Plan International Bénin, Cotonou, Bénin; ${ }^{7}$ Programme Santé de Lutte contre le Sida (PSLS), Cotonou, Bénin; ${ }^{8}$ Faculté des sciences de la santé, Université d'Abomey-Calavi, Cotonou, Bénin; ${ }^{9}$ Centre national hospitalier universitaire HMK de Cotonou, Cotonou, Bénin; ${ }^{10}$ Département de médecine sociale et préventive, Université Laval, Québec, Québec, Canada; ${ }^{11}$ Institut national de santé publique du Québec, Québec, Canada
\end{abstract}

\subsection{6/sextrans-2021-sti.248}

Background Men who have sex with men (MSM) are disproportionally affected by hepatitis worldwide despite the availability of safe and effective vaccines for hepatitis A and B. The aim of this analysis was to estimate the prevalence and risk factors for current and lifetime hepatitis B, and HCV antibodies among MSM in Cotonou.

Methods A two-stage cluster sampling procedure was used to recruit 204 MSM in the community-based PrEP demonstration study. First, 7 out of the 13 boroughs in Cotonou were selected using a probability proportional to size method and a fixed number of MSM were then randomly selected from each borough using a random route sampling procedure. A rapid immuno-chromatographic test was used for detecting HBV surface antigen (HBsAg, active 